\section{Hemorrhagic cystitis: A successful outcome for a challenging complication in stem cell transplant}

\author{
Sergio Pinzón Mariño, ${ }^{1}$ Samira Bakali \\ Badesa, ${ }^{2}$ María Jesús Viso Soriano, ${ }^{2}$ \\ Isabel Izquierdo Garcia ${ }^{1}$ \\ ${ }^{1}$ Department of Hematology and \\ Hemotherapy; ${ }^{2}$ Department of \\ Anatomical Pathology, Hospital \\ Universitario Miguel Servet, Spain
}

\begin{abstract}
Hemorrhagic cystitis (HC) secondary to $\mathrm{BK}$ polyomavirus (BKPyV) is a frequent complication related to allogenic stem cell transplantation. With an important morbidity and mortality, this disease doesn't have a stablished standard treatment or prophylaxis strategies. At this moment, the supportive therapies approved to treat included hyperhydration, forced diuresis and transfusion support. Cidofovir is a nucleotide analog of deoxycytidine monophosphate against DNA viruses and it has been described for the treatment of BKPyV-HC, but at this moment, is not a front-line therapy. We report a successful case after the use of Cidofovir without Probenecid. No adverse effect was developed under the treatment, and after 4 weeks of treatment, the patient achieved an excellent response.
\end{abstract}

\section{Introduction}

Hemorrhagic cystitis (HC) secondary to BK polyomavirus (BKPyV) is a challenging complication in patients with hematologic disease undergoing hematopoietic stem cell transplant (HSCT), with a variable prevalence depending of several factors such as age, sex and previous treatments.

Clinically, it debuts with a symptomatic triad compose of cystitis, macroscopic hematuria and high viral load, and requires the exclusion of other diseases. In the literature and the different clinical guidelines, prophylaxis treatment or standard management has not been established.

Cidofovir is an analog of deoxycytidine monophosphate. ${ }^{1}$ In Europe, the use of cidofovir in The European Conference on Infections in Leukemia (ECIL) guidelines gives a recommendation grade CIII and it is only indicated for the treatment of CMV retinitis in adults with acquired immunodeficiency syndrome (AIDS) and without renal impairment, relegating its use as a medicine out of indication. ${ }^{2}$

The aim of this case report is to show a successful improvement after using cidofovir in alloHSCT patient with $\mathrm{HC}$ secondary to BKPyV.

\section{Case Report}

A 46-year-old male patient was diagnosed with acute monoblastic myeloblastic leukemia NMPM1 mutated. Induction chemotherapeutic treatment with cytarabine and idarubicin was performed plus two cycles of consolidation. Subsequently, an autologous transplant was performed. A bone marrow aspirate for reevaluation showed positive metabolic residual disease $(0.53 \%)$ by immunophenotype, and positive mutated NPM1 with 14.7 copies, diagnosing a relapse of his leukemia. It was decided to perform an allogeneic bone marrow transplantation of identical HLA sister, and he received prophylaxis to graft versus host disease (GVHD) with cyclosporine and short-term methotrexate. He evolved favorably until day 60 post-transplant, when he showed a cutaneous GVHD that it was resolved with corticosteroids and cyclosporine.

At day 82 post-transplant, he went to the emergency room with tenesmus and dysuria without fever and macroscopic hematuria. Antibiotic treatment was started without improvement and worsening with the presence of macroscopic hematuria.

PCR for BKPyV was performed showing $5.2 \quad \log _{10}$ copies/mL and $6 \quad \log _{10}$ copies $/ \mathrm{mL}$, and in urine cytology, decoy cells (Figure 1) were observed. Bacterial cultures, PCR for CMV and adenovirus were performed in blood and urine with negative results, excluding other infectious etiologies. The patient was assessed by the Urology service ruling out structural pathology of the genitourinary system. Finally, the patient was diagnosed of Hemorrhagic cystitis secondary to BKPyV.

Treatment with intense hydration, alkalization and forced diuresis was initiated, however, the symptoms worsened by the presence of macroscopic hematuria with clots, causing urinary obstruction and requiring instrumentation for evacuation. Renal function deterioration was observed, previously presenting creatinine of 1.19 $\mathrm{mg} / \mathrm{dL}$ reaching $1.63 \mathrm{mg} / \mathrm{dL}$. An increased copies of BK virus in serum was detected, showing $6 \log _{10}$ copies/mL and persistence of the same copies in urine.

After 3 weeks of hydration and no clinical improvement, it was decided to start
Correspondence: Sergio Felipe Pinzón Mariño, Avenida Cesareo Alierta number 10 right staircase - 5th floor - Door Centro, Spain.

Tel.: +34.671110162

E-mail: spinzon48@gmail.com

Key words: BK virus; Cidofovir; Hematopoietic stem cell transplantation; Virus-associated hemorrhagic cystitis.

Contributions: the authors contributed equally.

Conflict of interest: the authors declare no potential conflict of interest.

Ethics approval and consent to participate: The study of this patient was approved by the Clinical Research Ethics Committee of Aragón (CEICA).

Consent for publication: Consent for publication was given.

Availability of data and materials:Authors have all the data filled in a database.

Received for publication: 3 April 2020.

Revision received: 15 July 2020.

Accepted for publication: 15 October 2020

This work is licensed under a Creative Commons Attribution-NonCommercial 4.0 International License (CC BY-NC 4.0).

(C) Copyright: the Author(s), 2021

Licensee PAGEPress, Italy

Hematology Reports 2021; 13:8574

doi:10.4081/hr.2021.8574

treatment with cidofovir at low doses (dose of $1 \mathrm{mg} / \mathrm{kg}$ weekly). The patient received a total of 4 weekly doses without Probenecid. No nephrotoxicity or myelotoxicity was detected under this treatment, and the patient showed clinical improvement, disappearing the urinary symptoms after the first dose, with improvement in the laboratory findings and decrease in viremia (3 $\log _{10}$ copies $\left./ \mathrm{mL}\right)$, but with persistence of viruria $\left(6 \log _{10}\right.$ copies $\left./ \mathrm{mL}\right)$.

\section{Discussion}

Hemorrhagic cystitis is not an unusual complication in patients who receive a HSCT, with a high morbidity and long-term hospital stay. ${ }^{3}$ Approximately $16 \%$ of the patients develop this kind of complication, ${ }^{4}$ with an incidence between $7-54 \%$ in adults and most frequently after allogenic than auto-HSCT. $^{2}$ There are several etiological factors such as post radiotherapy, toxic cause secondary to cytostatic such as cyclophosphamide, busulfan, and etoposide, and viral cause like BKPyV, CMV, and adenovirus. ${ }^{2,5}$ 
The typical episode of $\mathrm{HC}$ usually occurs between 2 and 4 weeks after HSCT. ${ }^{5}$ Clinical manifestations consist in signs and symptoms related to cystitis like dysuria, tenesmus, lower abdominal pain related to hematuria and urinary blood clots. ${ }^{2}$

It is required the presence of these symptoms and plasma viral loads of BKPyV $>7 \log _{10}$ copies/mL to perform the $\mathrm{BKPyV}-\mathrm{HC}$ diagnosis. It is also important to take into account that viral loads between 3-4 $\log _{10}$ copies $/ \mathrm{mL}$ could be founded in two thirds of patients with BKPyV-HC, ${ }^{2}$ however the viruria could help to lead the diagnosis, but it is not a part of the diagnostic criteria and is not useful in the followup. ${ }^{6}$

The evidence for the management of BKPyV-HC is limited, and much of the published data consists of non-randomized case series and case reports. ${ }^{7}$ There are no prophylactic treatment approved to prevent BKPyV-HC, ${ }^{2}$ only supportive measures such as hyperhydration, bladder irrigation, transfusion support and symptomatic therapy. ${ }^{2,8}$ Specific antiviral prophylaxis is not available. ${ }^{2}$ Several alternative treatments

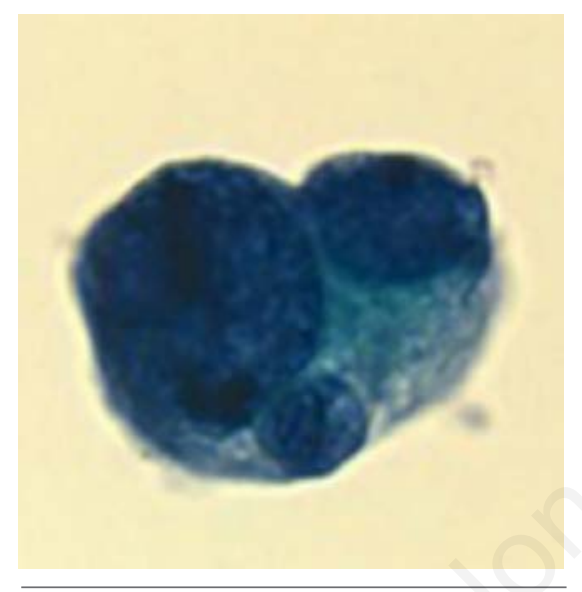

Figure 1. Decoy cells observed after cytology. have been studied, one of them is cidofovir which is an analog of deoxycytidine monophosphate with activity against herpesviruses and polyomaviruses, ${ }^{9}$ and is only indicated for the treatment of CMV retinitis in adults with acquired immunodeficiency and without renal impairment, relegating its use as a medicine out of indication. Also, in a recent study, the intravesicular instillation of cidofovir shows a clinical improvement in the $88 \%$ of the patients of this study. ${ }^{9}$ Exceptional access approval is required to use cidofovir in the treatment of $\mathrm{HC}$ secondary to BKPyV such as our patient.

\section{Conclusions}

$\mathrm{HC}$ secondary to BKPyV is a common and challenging complication in patients after HSCT without having a standard treatment. It is necessary to perform studies and clinical trials to improve the clinical evidence in this kind of patients. The use of intravenous cidofovir is controversial, but from this case report could be considered a safe alternative. It is necessary to perform clinical trials to prove this hypothesis.

\section{References}

1. Snoeck R, De Clercq E. Role of cidofovir in the treatment of DNA virus infections, other than CMV infections, in immunocompromised patients. Curr Opin Investig Drugs 2002;3:1561-6.

2. Cesaro S, Dalianis T, Hanssen Rinaldo $\mathrm{C}$, et al. ECIL guidelines for the prevention, diagnosis and treatment of BK polyomavirus-associated haemorrhagic cystitis in haematopoietic stem cell transplant recipients. J Antimicrob Chemother 2018;73:12-21.
3. Silva Lde P, Patah PA, Saliba RM, et al. Hemorrhagic cystitis after allogeneic hematopoietic stem cell transplants is the complex result of BK virus infection, preparative regimen intensity and donor type. Haematologica 2010;95:1183-90.

4. Lunde LE, Dasaraju S, Cao Q, et al. Hemorrhagic cystitis after allogeneic hematopoietic cell transplantation: risk factors, graft source and survival. Bone Marrow Transplant 2015;50:1432-7.

5. Colombel M, Labussière Wallet $\mathrm{H}$. Cistitis hemorrágica: fisiopatología y conducta práctica. EMC - Urología 2019;51:1-10.

6. Lee YJ, Zheng J, Kolitsopoulos Y, et al. Relationship of BK polyoma virus (BKV) in the urine with hemorrhagic cystitis and renal function in recipients of T Cell-depleted peripheral blood and cord blood stem cell transplantations. Biol Blood Marrow Transplant 2014;20:1204-10.

7. Aldiwani M, Tharakan T, Al-Hassani A, et al. BK Virus Associated Haemorrhagic Cystitis. A systematic review of current prevention and treatment strategies. Int J Surg 2019;63:3442.

8. Coomes EA, Wolfe Jacques A, Michelis $\mathrm{FV}$, et al. Efficacy of Cidofovir in Treatment of BK Virus-Induced Hemorrhagic Cystitis in Allogeneic Hematopoietic Cell Transplant Recipients. Biol Blood Marrow Transplant 2018;24:1901-5.

9. Tooker GM, Stafford KA, Nishioka J, et al. Intravesicular Cidofovir in the Treatment of BK Virus-Associated Hemorrhagic Cystitis Following Hematopoietic Stem Cell Transplantation. Ann Pharmacother 2020;54:547-53. 